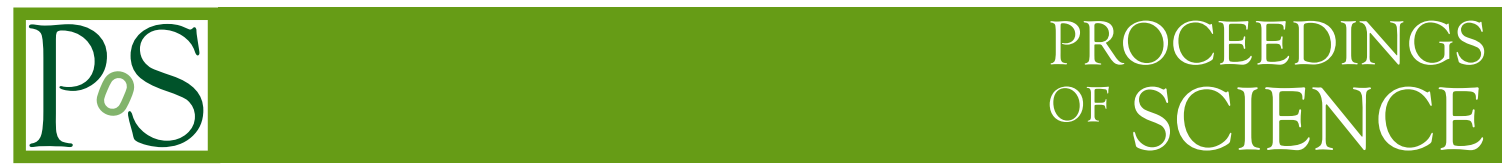

\title{
Critical fluctuations in QCD matter
}

\section{N. G. Antoniou*}

Faculty of Physics, University of Athens, Greece

E-mail: nantonio@phys.uoa.gr

The Ising-QCD effective action is introduced in order to study critical fluctuations in high-energy nuclear collisions. The order parameter in this description is the baryon-number density, a conserved quantity, compatible with the slow mode of the QCD critical point dynamics. We argue that finite-size scaling is the origin of critical fluctuations in the form of a fractal structure in the finite system. As a result, critical intermittency in nuclear collisions is a finite-size scaling phenomenon, linked to the QCD critical point, and may provide us with a route to observation of critical fluctuations in QCD matter.

Corfu Summer Institute 2017 "School and Workshops on Elementary Particle Physics and Gravity" 2-28 September 2017

Corfu, Greece

* Speaker. 


\section{Contents}

1. The QCD critical point (universality class; order parameter).

2. The Ising-QCD effective action.

3. Finite-size scaling; the origin of critical fluctuations.

4. Critical intermittency in nuclear collisions.

5. A route to observation of critical fluctuations.

6. Concluding remarks.

\section{The QCD critical point (universality class; order parameter)}

In the QCD phase diagram, the critical point (CP) is the endpoint of a first-order critical line $\left(\mu>\mu_{c}\right)$ and the starting point of an analytical crossover for $\mu<\mu_{c}$.

The value of the critical temperature, $T \simeq 160 \mathrm{MeV}$, is suggested by lattice QCD studies but the critical value of the chemical potential $\left(\mu_{c}\right)$ is very uncertain.

There is no consensus for the existence and location of the critical point, based on firstprinciple calculations (IQCD), therefore the role of the experimental search for critical fluctuations based on universality and scaling may become decisive. Complementary, crucial measurements of critical fluctuations are performed at present, in high-energy nuclear collisions, at BNL-RHIC (BES program) [1] and CERN-SPS (NA61 experiment) [2].

The phase transition at the QCD-CP (if it exists) is of second order and belongs to the $3 d$ Ising universality class $\left(\alpha \simeq 0, \beta \simeq \frac{1}{3}, \gamma \simeq \frac{4}{3}, v \simeq \frac{2}{3}, \delta \simeq 5\right.$ ).

The order parameter is a mixing between the chiral condensate ( $\sigma$-field) and the baryonnumber density $n_{b}[3]$.

The dynamics of the critical point selects the baryon density, the slow component of the order parameter, as the only thermodynamic quantity which may provide a self consistent description of critical fluctuations in QCD matter [4].

\section{The Ising-QCD effective action}

An effective potential for a scalar field theory in $3 d$ has been established on the basis of a Monte Carlo simulation of $3 d$ Ising system in an external field $h$ [5].

$$
\begin{gathered}
Z(\text { Ising })=\sum_{\left\{\varphi_{i}\right\}} \exp \left[\beta \sum_{i, j} \varphi_{i} \varphi_{j}+h \sum_{i} \varphi_{i}\right], \varphi_{i}= \pm 1 \\
S_{\text {eff }}(\text { scalar })=\int d^{3} \vec{x}\left[\frac{1}{2}|\nabla \varphi|^{2}+\frac{1}{2} m^{2} \varphi^{2}+g_{4} m \varphi^{4}+g_{6} \varphi^{\delta+1}-h \varphi\right]
\end{gathered}
$$

The effective action $S_{\text {eff }}$ is universal $\left(g_{4} \simeq 1, g_{6} \simeq 2\right)$ and describes the critical properties of any system belonging to $3 d$ Ising universality class, in terms of the scalar order parameter $\varphi$. The index $\delta$ is the isotherm critical exponent $(\delta \simeq 5$ ).

The QCD critical point is described by $S_{\text {eff }}$ (Ising-QCD) with $\varphi=\beta_{c}^{3} n_{b}$ and $h=\left(\mu-\mu_{c}\right) \beta_{c}$. The mass $m$ is linked to the correlation length $\xi$ and the corresponding critical exponent $v \simeq \frac{2}{3}$ : $m=\beta_{c} \xi^{-1}=\left(\frac{T-T_{c}}{T_{c}}\right)^{v}$. 
The partition function $Z=\int[\delta \varphi] \exp \left(-S_{e f f}\right)$ in the ensemble of constant configurations $(\nabla \varphi=$ $0)$ takes the form of a grand-canonical expansion:

$$
Z=\sum_{N_{b}} \zeta^{N_{b}} \exp \left[-\frac{1}{2} t^{2 v} \frac{N_{b}^{2}}{V}-g_{4} t \frac{N_{b}^{4}}{V^{3}}-g_{6} \frac{N_{b}^{\delta+1}}{V^{\delta}}\right] ; T \geq T_{c}
$$

$V$ : the volume in units $\beta_{c}^{3}, \zeta=\exp \left(\frac{\mu-\mu_{c}}{T_{c}}\right), t=\frac{T-T_{c}}{T_{c}}$ and $N_{b}$ the global baryon-number multiplicity [6].

In the Ising-QCD partition function one has incorporated the ingredients needed in order to describe dynamical fluctuations near the QCD critical point on the basis of scaling theory and universality. In particular, the universal form of $Z$ with the involvement of two fundamental indices $\left(v, q=\frac{d_{F}}{d}\right)$ may lead to a complete set of critical exponents:

$$
\alpha=2-v d, \beta=v d(1-q), \gamma=v d(2 q-1), \delta=\frac{q}{1-q}
$$

which obey the scaling laws and are linked to the Ising-QCD universality class if $v \simeq \frac{2}{3}, q \simeq \frac{5}{6}$.

\section{Finite-size scaling; the origin of critical fluctuations}

The general theory of finite-size scaling in critical systems predicts, at the critical point $(t=0$, $\zeta=1)$ the behaviour of QCD matter [7]:

(a) baryon-number susceptibility : $\chi_{b}(L) \sim L^{\gamma / v}$

(b) baryon-number density : $n_{b}(L) \sim L^{-\beta / v}$

in terms of the linear size $L$. The Ising-QCD prediction is compatible with this basic result:

$$
\begin{gathered}
Z=\sum_{N_{b}} \exp \left(-g_{6} \frac{N_{b}^{\delta+1}}{V^{\delta}}\right) \rightarrow\left\langle n_{b}\right\rangle \sim L^{d_{F}-1} ; d_{F}=1-\frac{\beta}{v} \\
\chi_{b}(L)=\frac{1}{V}\left[\left\langle N_{b}^{2}\right\rangle-\left\langle N_{b}\right\rangle^{2}\right] \rightarrow \chi_{b}(L) \sim L^{d(2 q-1)} ; 2 q-1=\frac{\gamma}{v d}
\end{gathered}
$$

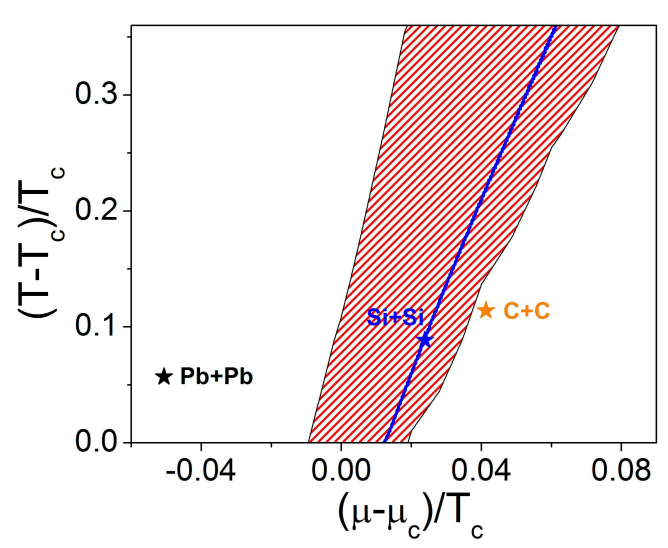

Figure 1: Description of the critical region and reveals the nature of the global critical fluctuations in the form of a monofractal structure:

$$
\left\langle N^{\kappa}\right\rangle \sim L^{\kappa d_{F}} ; d_{F}=1-\frac{\beta}{v}, \kappa=1,2, \ldots
$$

Departing from the critical point, these fluctuations are suppressed and with the help of the Ising-QCD partition function for $t \neq 0$, $\zeta \neq 1$ we may locate the critical region with the constraint: 


$$
\langle N\rangle \sim V^{\hat{q}} \text { with } \frac{3}{4}<\hat{q}<1
$$

The exponent $3 / 4$ corresponds to the dominance of the $\varphi^{4}$ term in the effective action, over the Ising behaviour $\sim \varphi^{6}$, in a distance from $t=0$, along the temperature direction (figure 1). The critical region is narrow along the chemical potential: For $T=T_{c} \rightarrow \frac{\delta \mu_{b}}{T_{c}} \simeq 0.03$ [6].

\section{Critical intermittency in nuclear collisions}

The fractal structure of critical fluctuations, $\left\langle N_{b}\right\rangle \sim L^{d_{F}}$, implies correlations at scales close to the correlation length $\xi>L$, of the form:

$$
\left\langle N_{b}\right\rangle \sim L^{d_{F}} \rightarrow\left\langle n_{b}(\vec{x}) n_{b}\left(\vec{x}^{\prime}\right)\right\rangle \sim\left|\vec{x}-\vec{x}^{\prime}\right|^{d_{F}-3} ; d_{F} \simeq \frac{5}{2}
$$

In transverse momentum space (in central rapidity region) a $2 d$ fractal with $\hat{d}_{F}=2\left(1-\frac{d_{F}}{d}\right)$ is developed, generating local correlations of the form

$$
\left\langle n_{b}(\vec{k}) n_{b}\left(\vec{k}^{\prime}\right)\right\rangle \sim\left|\vec{k}-\vec{k}^{\prime}\right|^{-\frac{2 d_{F}}{3}}
$$

These strong fluctuations in momentum space (locally) become observable in the phenomenon of intermittency [8].

The factorial moments in multiparticle production reveal the phenomenon of intermittency (dynamical local fluctuations) in the form of a power-law behaviour (we consider the second moment $F_{2}$ in $2 d$ ):

$$
F_{2}=\frac{\sum_{m}\left\langle n_{m}\left(n_{m}-1\right)\right\rangle}{\sum_{m}\left\langle n_{m}\right\rangle^{2}} \Rightarrow F_{2}(M) \sim\left(M^{2}\right)^{\varphi_{2}}
$$

for $M^{2} \gg 1\left(M^{2}\right.$ : number of $2 d$ cells). The exponent $\varphi_{2}$ (observable) is a characteristic intermittency index. The phenomenon of $2 d$ intermittency implies power-law correlations of the form:

$$
\left\langle n_{b}(\vec{k}) n_{b}\left(\vec{k}^{\prime}\right)\right\rangle \sim\left|\vec{k}-\vec{k}^{\prime}\right|^{-2 \varphi_{2}}
$$

It reveals fluctuations linked to the QCD critical point (critical intermittency) if $\varphi_{2}=\frac{d_{F}}{3}=q$ (the intermittency index $\varphi_{2}$ is equal to the critical exponent $q=\frac{5}{6}$ ).

\section{A route to observation of critical fluctuations}

(a) measurements of the intermittency index $\varphi_{2}$.

(b) If $\frac{3}{4}<\varphi_{2}<1 \rightarrow$ indication for critical fluctuations (the critical point corresponds to the value $\left.\varphi_{2}=\frac{5}{6}\right)$.

(c) Measurement of chemical freeze-out states leads to the location in the critical region (figure 1).

(d) A pilot measurement in $S_{i}+S_{i}$ at the SPS (NA49 experiment) has given $\varphi_{2} \simeq 0.96$ (the whole scheme is compatible with $T_{c} \simeq 150 \mathrm{MeV}, \mu_{c} \simeq 256 \mathrm{MeV}$ ) [8, 9]. 
The method is applicable to current experiments at CERN and BNL (SPS-NA61, RHIC-BES) in collisions of heavy and medium or small size nuclei:

I. $\mathrm{Pb}+\mathrm{Pb}, \mathrm{Au}+\mathrm{Au}$ : The crucial energy range for these processes at $\mathrm{CERN}(\mathrm{Pb}+\mathrm{Pb})$ and $\mathrm{BNL}$ $(\mathrm{Au}+\mathrm{Au})$ is:

$\mathrm{Pb}+\mathrm{Pb}$ at $12.3 \mathrm{GeV}<\sqrt{s_{N N}}<17.2 \mathrm{GeV}$ (SPS-NA61, beyond 2020)

$\mathrm{Au}+\mathrm{Au}$ at $14.5 \mathrm{GeV}<\sqrt{s_{N N}}<19.6 \mathrm{GeV}$ (RHIC-BES)

II. Be+Be, Ar+Sc, Xe+La at the highest SPS energy $\sqrt{s_{N N}} \simeq 17.2 \mathrm{GeV}$ (SPS-NA61)

\section{Concluding remarks}

A construction of Ising-QCD effective action was proposed, for the study of critical fluctuations in QCD matter.

A description of the QCD critical point in terms of baryon-number density, as the appropriate order parameter, was presented, compatible with universality and scaling theory.

In particular, the phenomenon of finite-size scaling was revealed, linked to the fundamental critical exponents $D_{h}=q d, D_{t}=\frac{1}{v}$, which are incorporated ih the Ising-QCD effective action.

It was emphasized that finite-size scaling leads to global and local critical fluctuations in QCD matter, accessible to measurements.

In particular, the phenomenon of intermittency in transverse momenta, with a well prescribed exponent, was identified as a finite-size scaling phenomenon, linked to the fluctuations at the QCD critical point (critical intermittency) when observed in the net-proton system [10].

A critical region in the phase diagram $(\mu, T)$, extremely narrow in the $\mu$-direction was found $\left(\frac{\delta \mu}{T_{c}} \simeq 0.03\right)$ providing an efficient instrument for the location of the QCD critical point.

Finally, a route to observation was proposed, based on crucial intermittency measurements and chemical freeze-out studies, linked to current experiments at CERN (SPS-NA61) and BNL (RHIC-BES).

\section{References}

[1] X. Luo and N. Xu, Nucl. Sci. Tech. 28, 112 (2017)

[2] M. Gazdicki (for the NA61 Collaboration) J. Phys. G36, 064039 (2009)

[3] K. Fukushima, T. Hatsuda, Rep. Prog. Phys. 74, 014001 (2011)

[4] Y. Minami, Phys. Rev. D83, 094019 (2011)

[5] M. M. Tsypin, Phys. Rev. Lett. 73, 2015 (1994)

[6] N. G. Antoniou, F. K. Diakonos, X. N. Maintas, C. E. Tsagkarakis, Phys. Rev. D97, 034015 (2018)

[7] H. Meyer-Ortmanns, Rev. Mod. Phys. 68, 473 (1996)

[8] A. Bialas, R. Peshanski, Nucl. Phys. B273 (1986), B308, 857 (1988); N. G. Antoniou PoS (CORFU 2016) 052

[9] T. Anticic et al. NA49 Collaboration, Eur. Phys. J C75, 587 (2015)

[10] N. G. Antoniou, F. K. Diakonos, A. S. Kapoyannis, K. S. Kousouris, Phys. Rev. Lett. 97, 032002 (2006) 